\title{
FINITE-TIME BLOWUP FOR A COMPLEX GINZBURG-LANDAU EQUATION
}

\author{
Thierry Cazenave \\ Université Pierre et Marie Curie \& CNRS \\ Laboratoire Jacques-Louis Lions \\ B.C. 187, 4 place Jussieu \\ 75252 Paris Cedex 05, France \\ email address: thierry.cazenave@upmc.fr \\ Flávio Dickstein \\ Instituto de Matemática \\ Universidade Federal do Rio de Janeiro \\ Caixa Postal 68530 \\ 21944-970 Rio de Janeiro, R.J., Brazil \\ email address: flavio@labma.ufrj.br \\ Fred B. Weissler \\ Université Paris 13, Sorbonne Paris Cité \\ CNRS UMR 7539 LAGA \\ 99 Avenue J.-B. Clément \\ F-93430 Villetaneuse, France \\ email address: weissler@math.univ-paris13.fr
}

\begin{abstract}
We prove that negative energy solutions of the complex GinzburgLandau equation $e^{-i \theta} u_{t}=\Delta u+|u|^{\alpha} u$ blow up in finite time, where $\alpha>0$ and $-\pi / 2<\theta<\pi / 2$. For a fixed initial value $u(0)$, we obtain estimates of the blow-up time $T_{\max }^{\theta}$ as $\theta \rightarrow \pm \pi / 2$. It turns out that $T_{\max }^{\theta}$ stays bounded (respectively, goes to infinity) as $\theta \rightarrow \pm \pi / 2$ in the case where the solution of the limiting nonlinear Schrödinger equation blows up in finite time (respectively, is global).
\end{abstract}

\section{INTRODUCTION}

This paper is concerned with the existence of solutions which blow up in finite time of the Cauchy problem

$$
\left\{\begin{array}{l}
e^{-i \theta} u_{t}=\Delta u+|u|^{\alpha} u \\
u(0)=u_{0}
\end{array}\right.
$$

in $\mathbb{R}^{N}$, where $\alpha>0$ and

$$
-\frac{\pi}{2} \leq \theta \leq \frac{\pi}{2}
$$

2010 Mathematics Subject Classification. 35Q56, 35B44.

Key words and phrases. Complex Ginzburg-Landau equation, finite time blowup, energy, variance.

Research supported by the "Brazilian-French Network in Mathematics".

Flávio Dickstein was partially supported by CNPq (Brasil) and by a "Research in Paris" grant from the City of Paris.

Fred B. Weissler benefited from a sabbatical leave (CRCT) from the University of Paris 13. 
More precisely, we seek conditions on the initial value $u_{0}$ which guarantee that the resulting solution is non-global. In addition, we wish to obtain estimates on the blow-up time, for a given initial value $u_{0}$, as a function of $\theta$.

Equation (GL) with $\theta=0$ reduces to the well known nonlinear heat equation $u_{t}-\Delta u=|u|^{\alpha} u$. For $\theta= \pm \pi / 2$, equation (GL) becomes the equally well known nonlinear Schrödinger equation $\pm i u_{t}+\Delta u+|u|^{\alpha} u=0$. Thus we see that (GL) is "intermediate" between the nonlinear heat and Schrödinger equations. Our overall objective is to understand finite time blowup of solutions of (GL) from a unified point of view, for all $-\pi / 2 \leq \theta \leq \pi / 2$.

The equation (GL) is a particular case of the more general complex GinzburgLandau equation

$$
u_{t}=e^{i \theta} \Delta u+e^{i \gamma}|u|^{\alpha} u .
$$

Equation (1.1) has been studied in the context of a wide variety of applications. For example, the nonlinear Schrödinger equation (i.e. (1.1) with $\theta=\gamma= \pm \pi / 2$ ) is an important model in nonlinear optics and in the study of weakly nonlinear dispersive waves. We refer the reader to the monograph [29] which has an extensive discussion of these and other applications. The nonlinear heat equation (i.e. (1.1) with $\theta=$ $\gamma=0$ ), often with a more general nonlinear term, is also an important model, in particular in biology and chemistry. We refer the reader to the monograph [5] for a sampling of such applications. In the more general case, equation (1.1) is used to model such phenomena as superconductivity, chemical turbulence and various types of fluid flows. See [3] and the references cited therein. A key feature associated to the phenomena modeled by (1.1) is the development of singularities. Solutions of (1.1) may be global in time or may cease to exist at some finite (blow-up) time. The existence of blowing-up solutions may be interpreted as the appearance of instabilities in the various applications of (1.1).

Local and global existence of solutions of (1.1), on both $\mathbb{R}^{N}$ and a domain $\Omega \subset \mathbb{R}^{N}$, are known under various boundary conditions and assumptions on the parameters, see e.g. $[4,7,8,15,20,21,22,23]$. On the other hand, there are relatively few results concerning the existence of solutions of (1.1) for which finitetime blowup occurs. In [31], blowing-up solutions for the equation (1.1) on $\mathbb{R}^{N}$ are proved to exist, when the equation is "close" to the nonlinear heat equation $u_{t}=\Delta u+|u|^{\alpha} u$, i.e. when $\theta=0$ and $|\gamma|$ is small. A result in the same spirit is obtained in [27] when the equation is "close" to the nonlinear Schrödinger equation $i u_{t}+\Delta u+|u|^{\alpha} u=0$. The result in [31] was significantly extended in [14], where the authors give a rigorous justification of the numerical and formal arguments of $[25,26]$. More precisely, they consider the equation (1.1) on $\mathbb{R}^{N}$ with $-\pi / 2<$ $\theta, \gamma<\pi / 2$ and prove the existence of blowing-up solutions when $\tan ^{2} \gamma+(\alpha+$ $2) \tan \gamma \tan \theta<\alpha+1$. Note also that, under certain assumptions on the parameters, blowup for an equation similar to (1.1) on a bounded domain with Dirichlet or periodic boundary conditions, but with the nonlinearity $|u|^{\alpha+1}$ instead of $|u|^{\alpha} u$ is proved to occur in $[16,17,24]$.

The equation (GL) has certain features not shared by the more general equation (1.1). First of all, stationary solutions of (GL) satisfy the same elliptic equation $\Delta u+|u|^{\alpha} u=0$, independent of the parameter $\theta$. Furthermore, and more significant for the present article, it turns out that its solutions satisfy energy identities similar to those satisfied by the solutions of the nonlinear heat and Schrödinger equations. 
See Proposition 2.3 below. Recall the energy functional is defined by

$$
E(w)=\frac{1}{2} \int_{\mathbb{R}^{N}}|\nabla w|^{2}-\frac{1}{\alpha+2} \int_{\mathbb{R}^{N}}|w|^{\alpha+2},
$$

for $w \in C_{0}\left(\mathbb{R}^{N}\right) \cap H^{1}\left(\mathbb{R}^{N}\right)$. This property was exploited in [28], where the authors apply Levine's argument [13] (see also [1]) and prove finite-time blowup of all negative energy solutions when $N=1,2, \alpha=2$ and $|\theta|<\pi / 4$. The calculations of [28] can be carried out for more general values of $\alpha$, and the condition $|\theta|<\pi / 4$ takes the form $\cos ^{2} \theta>\frac{2}{\alpha+2}$.

Our first main result is that if the initial value $u_{0}$ has negative energy and $-\pi / 2<\theta<\pi / 2$, then the corresponding solution of (GL) blows up in finite time. We make no assumption on $\alpha>0$. We essentially follow the energy method of [13]. The improvement with respect to [28], where a condition on $\alpha$ and $\theta$ appears, is due to the use of the identity (2.5) below.

Theorem 1.1. Suppose

$$
-\frac{\pi}{2}<\theta<\frac{\pi}{2}
$$

let $u_{0} \in C_{0}\left(\mathbb{R}^{N}\right) \cap H^{1}\left(\mathbb{R}^{N}\right)$ and let $u \in C\left(\left[0, T_{\max }\right), C_{0}\left(\mathbb{R}^{N}\right) \cap \mathbb{R}^{N}\right)$ be the corresponding maximal solution of $(\mathrm{GL})$. If $E\left(u_{0}\right)<0$, then $u$ blows up in finite time. More precisely,

$$
T_{\max } \leq \frac{\left\|u_{0}\right\|_{L^{2}}^{2}}{\alpha(\alpha+2)\left(-E\left(u_{0}\right)\right) \cos \theta} .
$$

Of course, $E\left(u_{0}\right)$ in the statement of Theorem 1.1 refers to the energy functional defined by (1.2). Theorem 1.1 shows that any solution of (GL) with negative initial energy blows up in finite time provided (1.3) holds. This raises the question of the behavior of the blow-up time as $\theta$ approaches $\pm \pi / 2$. Indeed, recall that the Cauchy problem for the nonlinear Schrödinger equation, i.e. the equation (GL) with $\theta= \pm \pi / 2$ is locally well-posed in $H^{1}\left(\mathbb{R}^{N}\right)$ if $\alpha<4 /(N-2)$. (See $[6,11]$.) Moreover, if $\alpha<4 / N$ then all solutions are global (see [6]), while if $\alpha \geq 4 / N$ then some solutions blow up in finite time (see [9,32]). More precisely, if the initial value $u_{0} \in H^{1}\left(\mathbb{R}^{N}\right)$ with negative energy has finite variance (i.e. $\int|x|^{2}\left|u_{0}\right|^{2}<\infty$ ), then the solution blows up in finite time. The same conclusion holds if, instead of assuming that $u_{0}$ has finite variance, we assume that either $N=1$ and $\alpha=4$, or else $N \geq 2, u_{0}$ is radially symmetric and $\alpha \leq 4$, see $[18,19]$.

Fix an initial value $u_{0} \in C_{0}\left(\mathbb{R}^{N}\right) \cap H^{1}\left(\mathbb{R}^{N}\right)$ such that $E\left(u_{0}\right)<0$ and, given $\theta \in(-\pi / 2, \pi / 2)$, let $u^{\theta}$ be the corresponding solution of (GL), so that $u^{\theta}$ blows up in finite time by Theorem 1.1. If $\alpha<4 / N$, then the solution of (GL) for $\theta= \pm \pi / 2$ is global, so we may expect that the blow-up time of $u^{\theta}$ goes to infinity as $\theta \rightarrow \pm \pi / 2$. This is indeed the case, as the following result shows.

Theorem 1.2. Fix an initial value $u_{0} \in C_{0}\left(\mathbb{R}^{N}\right) \cap H^{1}\left(\mathbb{R}^{N}\right)$ and, for every $\theta$ satisfying (1.3), let $u^{\theta} \in C\left(\left[0, T_{\max }^{\theta}\right), C_{0}\left(\mathbb{R}^{N}\right) \cap H^{1}\left(\mathbb{R}^{N}\right)\right)$ denote the corresponding maximal solution of (GL). If

$$
0<\alpha<\frac{4}{N},
$$

then there exists a constant $c=c\left(N, \alpha,\left\|u_{0}\right\|_{L^{2}}, E\left(u_{0}\right)\right)>0$ such that

$$
T_{\max }^{\theta} \geq \frac{c}{\cos \theta},
$$

for all $-\frac{\pi}{2}<\theta<\frac{\pi}{2}$. 
Remark 1.3. Note that, under the assumptions of Theorem 1.2 and if, in addition, $E\left(u_{0}\right)<0$, there exist $c, C>0$ such that

$$
\frac{c}{\cos \theta} \leq T_{\max }^{\theta} \leq \frac{C}{\cos \theta}
$$

for all $-\pi / 2<\theta<\pi / 2$. This follows from (1.5) and (1.4).

Global existence for the nonlinear Schrödinger equation with $\alpha<4 / N$ follows from the conservation of charge and energy and Gagliardo-Nirenberg's inequality. Similarly, Theorem 1.2 follows from energy identities and Gagliardo-Nirenberg's inequality.

Remark 1.4. Theorems 1.1 and 1.2 are equally valid, with essentially the same proofs, for solutions of (GL) on a smooth domain $\Omega \subset \mathbb{R}^{N}$ with Dirichlet boundary conditions. Moreover, in the case of a bounded domain Ball's proof of finite time blowup [1] works equally well for (GL) with $-\pi / 2<\theta<\pi / 2$, using the energy identities in Section 2.

As observed above, if $\alpha \geq 4 / N$ then negative energy, finite variance solutions of the nonlinear Schrödinger equation blow up in finite time. Thus we may expect that the blow-up time of $u^{\theta}$ remains bounded as $\theta \rightarrow \pm \pi / 2$. We have the following result.

Theorem 1.5. Suppose

$$
N \geq 2, \quad \frac{4}{N} \leq \alpha \leq 4,
$$

and fix a radially symmetric initial value $u_{0} \in H^{1}\left(\mathbb{R}^{N}\right) \cap C_{0}\left(\mathbb{R}^{N}\right)$. Given any $\theta$ satisfying (1.3), let $u^{\theta} \in C\left(\left[0, T_{\max }^{\theta}\right), C_{0}\left(\mathbb{R}^{N}\right) \cap H^{1}\left(\mathbb{R}^{N}\right)\right)$ denote the corresponding maximal solution of $(\mathrm{GL})$. If $E\left(u_{0}\right)<0$, then there exists $\bar{T}<\infty$ such that $T_{\max }^{\theta} \leq \bar{T}$ for all $-\frac{\pi}{2}<\theta<\frac{\pi}{2}$.

Blowup for the equation (GL) with $-\frac{\pi}{2}<\theta<\frac{\pi}{2}$ (i.e. Theorem 1.1) is proved by an energy argument. On the other hand, blowup for the nonlinear Schrödinger equation is proved by a variance argument (or a similar argument for a truncated variance as in $[18,19])$. It turns out that for the equation (GL) there is also a variance identity (and a truncated variance identity as well), see formulas (7.1) and (5.2) below. By combining the information derived from the truncated variance identity with the energy identities, we are able to establish the uniform estimate of the blow-up time of Theorem 1.5. We mention that the conditions that $u_{0}$ be radially symmetric and that $\alpha \leq 4$ are necessary for the crucial estimate in our proof, see Section 6. We do not know if the conclusion of Theorem 1.5 is true without these hypotheses.

Note that the assumptions on $u_{0}$ in Theorem 1.5 are precisely those made by Ogawa and Tsutsumi in [18], where the authors eliminate the finite variance assumption of $[9,32]$. One might expect that, if we were willing to assume that $u_{0}$ has finite variance, then we would not need the assumptions that $\alpha \leq 4$ and that $u_{0}$ is radially symmetric. In this case, the proof would be based on the variance identity (7.1) rather than on the truncated variance identity (5.2). Unfortunately, in this case as well, and for apparently different reasons, the same conditions are necessary for the crucial estimate of this other proof. See Section 7.

The rest of this paper is organized as follows. In the next section, we recall the basic local well-posedness results for the Cauchy problem (GL) and establish the 
fundamental energy identities. Theorems 1.1, 1.2 and 1.5 are proved successively in Sections 3, 4 and 5. In Section 6 we comment on the obstacles to proving Theorem 1.5 under less restrictive hypotheses. In Section 7, we outline the proof which could be given of Theorem 1.5 under the additional assumption of finite variance and comment on the related hypotheses.

\section{The local Cauchy problem: $-\pi / 2<\theta<\pi / 2$}

The linear equation associated with (GL) is

$$
u_{t}=e^{i \theta} \Delta u \text {. }
$$

It is well known that the operator $e^{i \theta} \Delta$ with domain $H^{2}\left(\mathbb{R}^{N}\right)$ generates a semigroup of contractions $\left(\mathcal{T}_{\theta}(t)\right)_{t \geq 0}$ on $L^{2}\left(\mathbb{R}^{N}\right)$. Moreover, since (1.3) holds, the semigroup $\left(\mathcal{T}_{\theta}(t)\right)_{t \geq 0}$ is analytic. Indeed, the semigroup $e^{z \Delta}$ is analytic in the half plane $\Re z>0$. In particular, $\mathcal{T}_{\theta}(t) \psi=G_{\theta}(t) \star \psi$, where the kernel $G_{\theta}(t)$ is defined by

$$
G_{\theta}(t)(x) \equiv\left(4 \pi t e^{i \theta}\right)^{-\frac{N}{2}} e^{-\frac{|x|^{2}}{4 t e^{i \theta}}} .
$$

Since

it follows that

$$
\left|G_{\theta}(t)(x)\right|=(4 \pi t)^{-\frac{N}{2}} e^{-\frac{|x|^{2} \cos \theta}{4 t}},
$$

$$
\left\|G_{\theta}(t)\right\|_{L^{\sigma}}= \begin{cases}\sigma^{-\frac{N}{2 \sigma}}(4 \pi t)^{-\frac{N}{2}\left(1-\frac{1}{\sigma}\right)}(\cos \theta)^{-\frac{N}{2 \sigma}} & \text { if } 1 \leq \sigma<\infty \\ (4 \pi t)^{-\frac{N}{2}} & \text { if } \sigma=\infty\end{cases}
$$

We deduce from (2.1) and Young's inequality that

$$
\left\|\mathcal{T}_{\theta}(t) \psi\right\|_{L^{r}} \leq(\cos \theta)^{-\frac{N}{2}\left(1-\frac{1}{p}+\frac{1}{r}\right)} t^{-\frac{N}{2}\left(\frac{1}{p}-\frac{1}{r}\right)}\|\psi\|_{L^{p}}
$$

for $1 \leq p \leq r \leq \infty$ and $\theta$ satisfying (1.3). It follows easily from (2.2) that $\left(\mathcal{T}_{\theta}(t)\right)_{t \geq 0}$ is a bounded $C_{0}$ semigroup on $L^{p}\left(\mathbb{R}^{N}\right)$ for $1 \leq p<\infty$ and on $C_{0}\left(\mathbb{R}^{N}\right)$.

It is immediate by a contraction mapping argument that the Cauchy problem $(\mathrm{GL})$ is locally well posed in $C_{0}\left(\mathbb{R}^{N}\right)$. Moreover, it is easy to see using the estimates (2.2) that $C_{0}\left(\mathbb{R}^{N}\right) \cap H^{1}\left(\mathbb{R}^{N}\right)$ is preserved under the action of (GL). More precisely, we have the following result.

Proposition 2.1. Suppose (1.3). Given any $u_{0} \in C_{0}\left(\mathbb{R}^{N}\right) \cap H^{1}\left(\mathbb{R}^{N}\right)$, there exist $T>0$ and a unique function $u \in C\left([0, T], C_{0}\left(\mathbb{R}^{N}\right) \cap H^{1}\left(\mathbb{R}^{N}\right)\right) \cap C\left((0, T), H^{2}\left(\mathbb{R}^{N}\right)\right) \cap$ $C^{1}\left((0, T), L^{2}\left(\mathbb{R}^{N}\right)\right)$ which satisfies $(\mathrm{GL})$ for all $t \in(0, T)$ and such that $u(0)=u_{0}$. Moreover, $u$ can be extended to a maximal interval [0, $\left.T_{\max }\right)$, and if $T_{\max }<\infty$ then $\|u(t)\|_{L^{\infty}} \rightarrow \infty$ as $t \uparrow T_{\max }$.

Remark 2.2. Let $u_{0} \in C_{0}\left(\mathbb{R}^{N}\right) \cap H^{1}\left(\mathbb{R}^{N}\right)$ and let $u$ be the corresponding solution of (GL) defined on the maximal interval $\left[0, T_{\max }\right.$ ), and given by Proposition 2.1. If, in addition, $\alpha<4 / N$, then (GL) is locally well posed in $L^{2}\left(\mathbb{R}^{N}\right)$ (see [30]). It is not difficult to show using the estimates (2.2) that the maximal existence times in $C_{0}\left(\mathbb{R}^{N}\right)$ and $L^{2}\left(\mathbb{R}^{N}\right)$ are the same; and so if $T_{\max }<\infty$, then $\|u(t)\|_{L^{2}} \rightarrow \infty$ as $t \uparrow T_{\max }$.

We collect below the energy identities that we use in the next sections.

Proposition 2.3. Suppose (1.3) and let $u_{0} \in C_{0}\left(\mathbb{R}^{N}\right) \cap H^{1}\left(\mathbb{R}^{N}\right)$. If $u$ is the corresponding solution of (GL) given by Proposition 2.1 and defined on the maximal interval $\left[0, T_{\max }\right)$, then the following properties hold. 
(i) Let the energy functional $E$ be defined by (1.2). It follows that

$$
\cos \theta \int_{s}^{t} \int_{\mathbb{R}^{N}}\left|u_{t}\right|^{2}+E(u(t))=E(u(s))
$$

for all $0 \leq s<t<T_{\max }$.

(ii) Set

$$
I(w)=\int_{\mathbb{R}^{N}}|\nabla w|^{2}-\int_{\mathbb{R}^{N}}|w|^{\alpha+2},
$$

for $w \in C_{0}\left(\mathbb{R}^{N}\right) \cap H^{1}\left(\mathbb{R}^{N}\right)$. It follows that

$$
\left|\int_{\mathbb{R}^{N}} u_{t} \bar{u}\right|=|I(u)|
$$

and

$$
\frac{d}{d t} \int_{\mathbb{R}^{N}}|u|^{2}=-2 \cos \theta I(u)
$$

for all $0<t<T_{\max }$.

Proof. The identity (2.3) follows by multiplying the equation (GL) by $\bar{u}_{t}$, integrating by parts on $\mathbb{R}^{N}$ and taking the real part. Multiplying the equation (GL) by $e^{i \theta} \bar{u}$ and integrating by parts on $\mathbb{R}^{N}$, we obtain

$$
\int_{\mathbb{R}^{N}} u_{t} \bar{u}=-e^{i \theta} I(u)
$$

Identity (2.5) follows by taking the modulus of both sides of (2.7), while (2.6) follows by taking the real part.

Remark 2.4. It follows easily from (2.6),(2.4) and (2.3) that

$$
\frac{d}{d t} \int_{\mathbb{R}^{N}}|u|^{2}=\frac{2 \alpha}{\alpha+2} \cos \theta \int_{\mathbb{R}^{N}}|u|^{\alpha+2}+4 \cos ^{2} \theta \int_{0}^{t} \int_{\mathbb{R}^{N}}\left|u_{t}\right|^{2}-4 \cos \theta E\left(u_{0}\right),
$$

and

$$
\begin{aligned}
\frac{d}{d t} \int_{\mathbb{R}^{N}}|u|^{2}=\alpha \cos \theta & \int_{\mathbb{R}^{N}}|\nabla u|^{2} \\
& +2(\alpha+2) \cos ^{2} \theta \int_{0}^{t} \int_{\mathbb{R}^{N}}\left|u_{t}\right|^{2}-2(\alpha+2) \cos \theta E\left(u_{0}\right) .
\end{aligned}
$$

\section{Proof of Theorem 1.1}

We use the argument of [10, pp. 185-186]. Note that, by $(2.3), E(u(t)) \leq E\left(u_{0}\right)<$ 0 for all $0 \leq t<T_{\max }$, so that

$$
\begin{aligned}
I(u(t)) & =(\alpha+2) E(u(t))-\frac{\alpha}{2} \int_{\mathbb{R}^{N}}|\nabla u(t)|^{2} \\
& \leq(\alpha+2) E(u(t)) \leq(\alpha+2) E\left(u_{0}\right)<0,
\end{aligned}
$$

for all $0<t<T_{\max }$. Set

$$
f(t)=\|u(t)\|_{L^{2}}^{2}, \quad e(t)=E(u(t)) .
$$

We deduce from (2.3) that

$$
\frac{d e}{d t}=-\cos \theta\left\|u_{t}\right\|_{L^{2}}^{2} \leq 0,
$$


and from (2.6) and (3.1) that

$$
\frac{d f}{d t}=-2 \cos \theta I(u(t))>0 .
$$

It follows from (3.2) and the Cauchy-Schwarz inequality that

$$
-f \frac{d e}{d t}=f \cos \theta\left\|u_{t}\right\|_{L^{2}}^{2}=\cos \theta\|u\|_{L^{2}}^{2}\left\|u_{t}\right\|_{L^{2}}^{2} \geq \cos \theta\left|\int_{\mathbb{R}^{N}} u_{t} \bar{u}\right|^{2} .
$$

Using (2.5) and (3.3), we deduce that

$$
\begin{aligned}
-f \frac{d e}{d t} & \geq \cos \theta(I(u(t)))^{2}=(-I(u(t)))(-\cos \theta I(u(t))) \\
& =\frac{1}{2}(-I(u(t))) \frac{d f}{d t} \geq \frac{\alpha+2}{2}(-e) \frac{d f}{d t} .
\end{aligned}
$$

This means that

$$
\frac{d}{d t}\left(-e f^{-\frac{\alpha+2}{2}}\right) \geq 0
$$

so that

$$
-e \geq \eta f^{\frac{\alpha+2}{2}}
$$

where

$$
\eta=\left(-E\left(u_{0}\right)\right)\left\|u_{0}\right\|_{L^{2}}^{-(\alpha+2)}
$$

It follows from (3.3), (3.1) and (3.5) that

$$
\frac{d f}{d t} \geq 2(\alpha+2)(\cos \theta)(-e) \geq 2 \eta(\alpha+2)(\cos \theta) f^{\frac{\alpha+2}{2}},
$$

so that

$$
\frac{d}{d t}\left[\eta \alpha(\alpha+2)(\cos \theta) t+f^{-\frac{\alpha}{2}}\right] \leq 0
$$

Integrating (3.7) between 0 and $t \in\left(0, T_{\max }\right)$, and applying (3.6), we deduce that

$$
t \leq \frac{\left\|u_{0}\right\|_{L^{2}}^{2}}{\alpha(\alpha+2)\left(-E\left(u_{0}\right)\right) \cos \theta},
$$

for all $0<t<T_{\max }$. The result follows by letting $t \uparrow T_{\max }$.

\section{Proof of Theorem 1.2}

We first note that by Gagliardo-Nirenberg's inequality there exists $c=c(N)$ such that

$$
\int_{\mathbb{R}^{N}}|u|^{2+\frac{4}{N}} \leq c \int_{\mathbb{R}^{N}}|\nabla u|^{2}\left(\int_{\mathbb{R}^{N}}|u|^{2}\right)^{\frac{2}{N}}
$$

for all $u \in H^{1}\left(\mathbb{R}^{N}\right)$. Applying Hölder's inequality and (4.1), we deduce that

$$
\begin{aligned}
\int_{\mathbb{R}^{N}}|u|^{\alpha+2} & \leq\left(\int_{\mathbb{R}^{N}}|u|^{2+\frac{4}{N}}\right)^{\frac{N \alpha}{4}}\left(\int_{\mathbb{R}^{N}}|u|^{2}\right)^{\frac{4-N \alpha}{4}} \\
& \leq c^{\frac{N \alpha}{4}}\|\nabla u\|_{L^{2}}^{\frac{N \alpha}{2}}\|u\|_{L^{2}}^{\frac{4-(N-2) \alpha}{2}} .
\end{aligned}
$$

We now use Young's inequality

$$
x y \leq \frac{N \alpha}{4} \varepsilon^{\frac{4}{N \alpha}} x^{\frac{4}{N \alpha}}+\frac{4-N \alpha}{4} \varepsilon^{-\frac{4}{4-N \alpha}} y^{\frac{4}{4-N \alpha}},
$$

with

$$
\varepsilon=\left(\frac{\alpha+2}{N \alpha c}\right)^{\frac{N \alpha}{4}}
$$


and we obtain

$$
\begin{aligned}
\frac{1}{\alpha+2} \int_{\mathbb{R}^{N}}|u|^{\alpha+2} & \leq \frac{1}{4}\|\nabla u\|_{L^{2}}^{2}+\frac{4-N \alpha}{4(\alpha+2)}\left(\frac{N \alpha c}{\alpha+2}\right)^{\frac{N \alpha}{4-N \alpha}}\|u\|_{L^{2}}^{\frac{2[4-(N-2) \alpha]}{4-N \alpha}} \\
& \leq \frac{1}{4}\|\nabla u\|_{L^{2}}^{2}+(N c)^{\frac{N \alpha}{4-N \alpha}}\|u\|_{L^{2}}^{\frac{2[4-(N-2) \alpha]}{4-N \alpha}}
\end{aligned}
$$

so that

$$
\frac{1}{\alpha+2} \int_{\mathbb{R}^{N}}|u|^{\alpha+2} \leq \frac{1}{4}\|\nabla u\|_{L^{2}}^{2}+\left[(N c)^{N \alpha}\|u\|_{L^{2}}^{2[4-(N-2) \alpha]}\right]^{\frac{1}{4-N \alpha}} .
$$

We now prove (1.5). If $T_{\max }^{\theta}=\infty$, there is nothing to prove. We then assume $T_{\max }^{\theta}<\infty$, so that

$$
\left\|u^{\theta}(t)\right\|_{L^{2}} \uparrow \infty \quad \text { as } \quad t \uparrow T_{\max }^{\theta}
$$

by Remark 2.2. Set

$$
S^{\theta}=\sup \left\{t \in\left[0, T_{\max }^{\theta}\right) ;\left\|u^{\theta}(s)\right\|_{L^{2}}^{2} \leq 2\left\|u_{0}\right\|_{L^{2}}^{2} \text { for } 0 \leq s \leq t\right\} .
$$

It follows from (4.4) that $S^{\theta}<T_{\max }^{\theta}$ and

$$
\left\|u^{\theta}\left(S^{\theta}\right)\right\|_{L^{2}}^{2}=2\left\|u_{0}\right\|_{L^{2}}^{2} .
$$

Since $E\left(u^{\theta}(t)\right) \leq E\left(u_{0}\right)$ by $(2.3)$ and

$$
\left\|u^{\theta}(t)\right\|_{L^{2}}^{2} \leq 2\left\|u_{0}\right\|_{L^{2}}^{2}
$$

for $0 \leq t \leq S^{\theta}$, it follows from (4.3) that

$$
\left\|\nabla u^{\theta}(t)\right\|_{L^{2}}^{2} \leq 4 E\left(u_{0}\right)+4 K^{\frac{1}{4-N \alpha}}
$$

where

$$
K=(N c)^{N \alpha}\left(2\left\|u_{0}\right\|_{L^{2}}^{2}\right)^{4-(N-2) \alpha} .
$$

Furthermore, (4.3), (4.6) and (4.7) imply

$$
\left\|u^{\theta}(t)\right\|_{L^{\alpha+2}}^{\alpha+2} \leq(\alpha+2) E\left(u_{0}\right)+2(\alpha+2) K^{\frac{1}{4-N \alpha}},
$$

so that

$$
\begin{aligned}
\left|I\left(u^{\theta}(t)\right)\right| & \leq \max \left\{\left\|\nabla u^{\theta}(t)\right\|_{L^{2}}^{2},\left\|u^{\theta}\right\|_{L^{\alpha+2}}^{\alpha+2}\right\} \\
& \leq(\alpha+4)\left[E\left(u_{0}\right)\right]^{+}+2(\alpha+2) K^{\frac{1}{4-N \alpha}}
\end{aligned}
$$

for $0 \leq t \leq S^{\theta}$. Applying (2.6) and (4.9), we deduce that

$$
\left\|u^{\theta}\left(S^{\theta}\right)\right\|_{L^{2}}^{2} \leq\left\|u_{0}\right\|_{L^{2}}^{2}+2(\cos \theta)\left[(\alpha+4)\left[E\left(u_{0}\right)\right]^{+}+2(\alpha+2) K^{\frac{1}{4-N \alpha}}\right] S^{\theta} .
$$

It now follows from (4.10) and (4.5) that

$$
S^{\theta} \geq \frac{\left\|u_{0}\right\|_{L^{2}}^{2}}{2\left[(\alpha+4)\left[E\left(u_{0}\right)\right]^{+}+2(\alpha+2) K^{\frac{1}{4-N \alpha}}\right] \cos \theta} .
$$

Since $T_{\max }^{\theta} \geq S^{\theta}$, the result follows from (4.11).

Remark 4.1. Suppose $E\left(u_{0}\right) \leq 0$. It follows from (4.11) that

$$
T_{\max }^{\theta} \geq \frac{\left\|u_{0}\right\|_{L^{2}}^{2}}{4(\alpha+2) K^{\frac{1}{4-N \alpha}} \cos \theta} .
$$


For a fixed $\theta$, the right-hand side converges to 0 very fast as $\alpha \uparrow 4 / N$, so the estimate is certainly not optimal with respect to the dependence on $\alpha$. Compare the estimate from above given in Remark 5.4.

\section{Proof of Theorem 1.5}

Our proof of Theorem 1.5 is modeled on the proof of finite time blowup for the nonlinear Schrödinger equation $([32,9,18])$. The basic idea is to estimate $\frac{d^{2}}{d t^{2}} \int \Psi(x)|u|^{2}$ for an appropriate function $\Psi>0$, in terms of the initial energy $E\left(u_{0}\right)$. If $E\left(u_{0}\right)<0$, this estimate implies that $\int \Psi(x)|u|^{2}$, becomes negative in finite time, thus showing that the solution cannot be global.

In the case of (GL), we have the following generalized variance identity.

Lemma 5.1. Fix a real-valued function $\Psi \in C^{\infty}\left(\mathbb{R}^{N}\right) \cap W^{4, \infty}\left(\mathbb{R}^{N}\right)$. Suppose (1.3), let $u_{0} \in C_{0}\left(\mathbb{R}^{N}\right) \cap H^{1}\left(\mathbb{R}^{N}\right)$ and consider the corresponding maximal solution $u \in$ $C\left(\left[0, T_{\max }\right), C_{0}\left(\mathbb{R}^{N}\right) \cap H^{1}\left(\mathbb{R}^{N}\right)\right)$ of (GL). It follows that the map $t \mapsto \int_{\mathbb{R}^{N}} \Psi|u|^{2}$ belongs to $C^{2}\left(\left[0, T_{\max }\right)\right)$,

$$
\begin{array}{r}
\frac{1}{2} \frac{d}{d t} \int_{\mathbb{R}^{N}} \Psi|u|^{2}=\cos \theta\left(-\int_{\mathbb{R}^{N}} \Psi|\nabla u|^{2}+\int_{\mathbb{R}^{N}} \Psi|u|^{\alpha+2}\right. \\
\left.+\frac{1}{2} \int_{\mathbb{R}^{N}} \Delta \Psi|u|^{2}\right) \\
+\sin \theta \Im \int_{\mathbb{R}^{N}} \nabla \Psi \bar{u} \nabla u,
\end{array}
$$

and

$$
\begin{aligned}
& \frac{1}{2} \frac{d^{2}}{d t^{2}} \int_{\mathbb{R}^{N}} \Psi|u|^{2}=-\frac{1}{2} \int_{\mathbb{R}^{N}} \Delta^{2} \Psi|u|^{2}-\frac{\alpha}{\alpha+2} \int_{\mathbb{R}^{N}} \Delta \Psi|u|^{\alpha+2} \\
& +2 \Re \int_{\mathbb{R}^{N}}\langle H(\Psi) \nabla \bar{u}, \nabla u\rangle+\cos \theta \frac{d}{d t} \int_{\mathbb{R}^{N}}\left\{-2 \Psi|\nabla u|^{2}+\frac{\alpha+4}{\alpha+2} \Psi|u|^{\alpha+2}+\Delta \Psi|u|^{2}\right\} \\
& -2 \cos ^{2} \theta \int_{\mathbb{R}^{N}} \Psi\left|u_{t}\right|^{2},
\end{aligned}
$$

for all $0 \leq t<T_{\max }$, where $H(\Psi)$ is the Hessian matrix $\left(\partial_{i j}^{2} \Psi\right)_{i, j}$.

Proof. Multiplying the equation (GL) by $e^{i \theta} \Psi(x) \bar{u}$, taking the real part and using the identity

$$
2 \Re(\nabla \Psi \bar{u} \nabla u)=\nabla \cdot\left(\nabla \Psi|u|^{2}\right)-\Delta \Psi|u|^{2},
$$

we obtain (5.1). We now differentiate (5.1) with respect to $t$. We begin with the term in factor of $\sin \theta$ and we note that, using the identity

$$
\nabla \Psi \bar{u} \nabla u_{t}=\nabla \cdot\left(\nabla \Psi u_{t} \bar{u}\right)-(\nabla \Psi \cdot \nabla \bar{u}) u_{t}-\Delta \Psi \bar{u} u_{t},
$$

and integration by parts,

$$
\frac{d}{d t}\left(\sin \theta \Im \int_{\mathbb{R}^{N}} \nabla \Psi \bar{u} \nabla u\right)=-\sin \theta\left(\Im \int_{\mathbb{R}^{N}} \Delta \Psi \bar{u} u_{t}+2 \Im \int_{\mathbb{R}^{N}}(\nabla \Psi \cdot \nabla \bar{u}) u_{t}\right) .
$$

i.e.

$$
\frac{d}{d t}\left(\sin \theta \Im \int_{\mathbb{R}^{N}} \nabla \Psi \bar{u} \nabla u\right)=-\sin \theta \Im \int_{\mathbb{R}^{N}}[\Delta \Psi \bar{u}+2 \nabla \Psi \cdot \nabla \bar{u}] u_{t}
$$


We rewrite this last identity in the form

$$
\begin{aligned}
\frac{d}{d t}\left(\sin \theta \Im \int_{\mathbb{R}^{N}} \nabla \Psi \bar{u} \nabla u\right)=\cos \theta \Re \int_{\mathbb{R}^{N}} & {[\Delta \Psi \bar{u}+2 \nabla \Psi \cdot \nabla \bar{u}] u_{t} } \\
& -\Re \int_{\mathbb{R}^{N}}[\Delta \Psi \bar{u}+2 \nabla \Psi \cdot \nabla \bar{u}] e^{-i \theta} u_{t} .
\end{aligned}
$$

Using (GL) and the identities

$$
\begin{gathered}
\Re(\nabla \Psi \cdot \nabla \bar{u})|u|^{\alpha} u=\frac{1}{\alpha+2} \nabla \cdot\left(\nabla \Psi|u|^{\alpha+2}\right)-\frac{1}{\alpha+2} \Delta \Psi|u|^{\alpha+2}, \\
\Re \nabla(\nabla \Psi \cdot \nabla \bar{u}) \cdot \nabla u=\frac{1}{2} \nabla \cdot\left(\nabla \Psi|\nabla u|^{2}\right)+\Re\langle H(\Psi) \nabla \bar{u}, \nabla u\rangle-\frac{1}{2} \Delta \Psi|\nabla u|^{2},
\end{gathered}
$$

we see that

$$
\begin{gathered}
-\Re \int_{\mathbb{R}^{N}}[\Delta \Psi \bar{u}+2 \nabla \Psi \cdot \nabla \bar{u}] e^{-i \theta} u_{t}=-\Re \int_{\mathbb{R}^{N}}[\Delta \Psi \bar{u}+2 \nabla \Psi \cdot \nabla \bar{u}]\left(\Delta u+|u|^{\alpha} u\right) \\
=-\frac{1}{2} \int_{\mathbb{R}^{N}} \Delta^{2} \Psi|u|^{2}-\frac{\alpha}{\alpha+2} \int_{\mathbb{R}^{N}} \Delta \Psi|u|^{\alpha+2}+2 \Re \int_{\mathbb{R}^{N}}\langle H(\Psi) \nabla \bar{u}, \nabla u\rangle .
\end{gathered}
$$

We now deduce from (5.3) and (5.4) that

$$
\begin{aligned}
& \frac{d}{d t}\left(\sin \theta \Im \int_{\mathbb{R}^{N}} \nabla \Psi \bar{u} \nabla u\right)= \\
&-\frac{1}{2} \int_{\mathbb{R}^{N}} \Delta^{2} \Psi|u|^{2}-\frac{\alpha}{\alpha+2} \int_{\mathbb{R}^{N}} \Delta \Psi|u|^{\alpha+2}+2 \Re \int_{\mathbb{R}^{N}}\langle H(\Psi) \nabla \bar{u}, \nabla u\rangle \\
&+\cos \theta \Re \int_{\mathbb{R}^{N}}[\Delta \Psi \bar{u}+2 \nabla \Psi \cdot \nabla \bar{u}] u_{t} .
\end{aligned}
$$

Note that

$$
\begin{aligned}
\frac{d}{d t} \int_{\mathbb{R}^{N}} \Psi\left(\frac{|\nabla u|^{2}}{2}-\frac{|u|^{\alpha+2}}{\alpha+2}\right) & =\Re \int_{\mathbb{R}^{N}} \Psi\left(\nabla \bar{u} \cdot \nabla u_{t}-|u|^{\alpha} \bar{u} u_{t}\right) \\
& =-\Re \int_{\mathbb{R}^{N}}\left[\left(\Psi\left(\Delta \bar{u}+|u|^{\alpha} \bar{u}\right) u_{t}\right)+(\nabla \Psi \cdot \nabla \bar{u}) u_{t}\right. \\
& =-\cos \theta \int_{\mathbb{R}^{N}} \Psi\left|u_{t}\right|^{2}-\Re \int_{\mathbb{R}^{N}}(\nabla \Psi \cdot \nabla \bar{u}) u_{t},
\end{aligned}
$$

so that

$$
2 \Re \int_{\mathbb{R}^{N}}(\nabla \Psi \cdot \nabla \bar{u}) u_{t}=-2 \cos \theta \int_{\mathbb{R}^{N}} \Psi\left|u_{t}\right|^{2}-\frac{d}{d t} \int_{\mathbb{R}^{N}}\left(\Psi|\nabla u|^{2}-\frac{2}{\alpha+2} \Psi|u|^{\alpha+2}\right) .
$$

Moreover,

$$
\Re \int_{\mathbb{R}^{N}} \Delta \Psi \bar{u} u_{t}=\frac{d}{d t} \frac{1}{2} \int_{\mathbb{R}^{N}} \Delta \Psi|u|^{2}
$$


We deduce from (5.5), (5.6) and (5.7) that

$$
\begin{aligned}
& \frac{d}{d t}\left(\sin \theta \Im \int_{\mathbb{R}^{N}} \nabla \Psi \bar{u} \nabla u\right) \\
&-\frac{1}{2} \int_{\mathbb{R}^{N}} \Delta^{2} \Psi|u|^{2}-\frac{\alpha}{\alpha+2} \int_{\mathbb{R}^{N}} \Delta \Psi|u|^{\alpha+2}+2 \Re \int_{\mathbb{R}^{N}}\langle H(\Psi) \nabla \bar{u}, \nabla u\rangle \\
& \quad+\cos \theta \frac{d}{d t} \int_{\mathbb{R}^{N}}\left(-\Psi|\nabla u|^{2}+\frac{2}{\alpha+2} \Psi|u|^{\alpha+2}+\frac{1}{2} \Delta \Psi|u|^{2}\right) \\
& \quad-2 \cos ^{2} \theta \int_{\mathbb{R}^{N}} \Psi\left|u_{t}\right|^{2} .
\end{aligned}
$$

Taking now the time-derivative of (5.1) and applying (5.8), we obtain (5.2).

The next tool we use for the proof of Theorem 1.5 is the following estimate. It says that the maximal existence time of a solution $u$ of (GL) is controlled, independently of $\theta$, by the maximal time until which $\|u(t)\|_{L^{2}}$ remains bounded by a (fixed) multiple of $\left\|u_{0}\right\|_{L^{2}}$.

Lemma 5.2. Suppose (1.3), let $u_{0} \in C_{0}\left(\mathbb{R}^{N}\right) \cap H^{1}\left(\mathbb{R}^{N}\right)$ and consider the corresponding maximal solution $u \in C\left(\left[0, T_{\max }\right), C_{0}\left(\mathbb{R}^{N}\right) \cap H^{1}\left(\mathbb{R}^{N}\right)\right)$ of $(\mathrm{GL})$. Set

$$
\tau=\sup \left\{t \in\left[0, T_{\max }\right) ;\|u(s)\|_{L^{2}}^{2} \leq K\left\|u_{0}\right\|_{L^{2}}^{2} \text { for } 0 \leq s \leq t\right\},
$$

where

$$
K=\left[1-\left(\frac{\alpha+4}{2 \alpha+4}\right)^{\frac{1}{2}}\right]^{-1}>1,
$$

so that $0 \leq \tau \leq T_{\max }$. If $E\left(u_{0}\right) \leq 0$, then $T_{\max } \leq \frac{\alpha+4}{\alpha} \tau$.

Proof. If $\tau=T_{\max }$, there is nothing to prove, so we now assume $\tau<T_{\max }$, so that

$$
\|u(t)\|_{L^{2}}^{2} \leq\|u(\tau)\|_{L^{2}}^{2}=K\left\|u_{0}\right\|_{L^{2}}^{2}, \quad 0 \leq t \leq \tau .
$$

Since $E\left(u_{0}\right) \leq 0$, it follows from (2.8) that the map $t \mapsto\|u(t)\|_{L^{2}}$ is nondecreasing on $\left[0, T_{\max }\right)$; and so, using $(5.11)$

$$
\|u(t)\|_{L^{2}}^{2} \geq K\left\|u_{0}\right\|_{L^{2}}^{2}, \quad \tau \leq t<T_{\max } .
$$

We now use calculations based on Levine [13]. We deduce from (2.9) that

$$
\frac{d}{d t} \int_{\mathbb{R}^{N}}|u|^{2} \geq 2(\alpha+2) \cos ^{2} \theta \int_{0}^{t} \int_{\mathbb{R}^{N}}\left|u_{t}\right|^{2}
$$

Set

$$
h(t)=\int_{0}^{t} \int_{\mathbb{R}^{N}}|u|^{2} .
$$

It follows from (5.13) and the Cauchy-Schwarz inequality that

$$
\begin{aligned}
{\left[2(\alpha+2) \cos ^{2} \theta\right]^{-1} h h^{\prime \prime} } & \geq h \int_{0}^{t} \int_{\mathbb{R}^{N}}\left|u_{t}\right|^{2} \geq\left(\int_{0}^{t} \int_{\mathbb{R}^{N}}|u|\left|u_{t}\right|\right)^{2} \\
& \geq\left(\int_{0}^{t}\left|\int_{\mathbb{R}^{N}} u_{t} \bar{u}\right|\right)^{2}
\end{aligned}
$$

Since $I(u(t)) \leq(\alpha+2) E(u(t)) \leq 0$ by (3.1), identities (2.5) and (2.6) yield

$$
\left|\int_{\mathbb{R}^{N}} u_{t} \bar{u}\right|=\frac{1}{2 \cos \theta} \frac{d}{d t} \int_{\mathbb{R}^{N}}|u|^{2}=\frac{1}{2 \cos \theta} h^{\prime \prime}(t) .
$$


We deduce from (5.15) and (5.16) that

$$
h h^{\prime \prime} \geq \frac{\alpha+2}{2}\left(h^{\prime}(t)-h^{\prime}(0)\right)^{2} .
$$

It follows from (5.17) and (5.12) that

$$
h h^{\prime \prime} \geq \frac{\alpha+2}{2}\left(\frac{K-1}{K}\right)^{2}\left[h^{\prime}(t)\right]^{2}=\frac{\alpha+4}{4}\left[h^{\prime}(t)\right]^{2},
$$

for all $\tau \leq t<T_{\max }$. This means that $\left(h^{-\frac{\alpha}{4}}\right)^{\prime \prime} \leq 0$ on $\left[\tau, T_{\max }\right)$; and so

$$
h(t)^{-\frac{\alpha}{4}} \leq h(\tau)^{-\frac{\alpha}{4}}+(t-\tau)\left(h^{-\frac{\alpha}{4}}\right)^{\prime}(\tau)=h(\tau)^{-\frac{\alpha}{4}}\left[1-\frac{\alpha}{4}(t-\tau) h(\tau)^{-1} h^{\prime}(\tau)\right],
$$

for $\tau \leq t \leq T_{\max }$. Since $h(t)^{-\frac{\alpha}{4}} \geq 0$, we deduce that for every $\tau \leq t<T_{\max }$,

i.e.

$$
\frac{\alpha}{4}(t-\tau) h(\tau)^{-1} h^{\prime}(\tau) \leq 1
$$

$$
(t-\tau)\|u(\tau)\|_{L^{2}}^{2} \leq \frac{4}{\alpha} \int_{0}^{\tau}\|u(s)\|_{L^{2}}^{2} d s \leq \frac{4}{\alpha} \tau\|u(\tau)\|_{L^{2}}^{2},
$$

where we used (5.11) in the last inequality. Thus $t \leq \frac{\alpha+4}{\alpha} \tau$ for all $\tau \leq t<T_{\max }$, which proves the desired inequality.

The last ingredient we use in the proof of Theorem 1.5 is Lemma 5.3 below. It is an estimate, based on Ogawa and Tsutsumi [18], which enables us to choose an appropriate function $\Psi$ in Lemma 5.1. Unfortunately, we have only been able to accomplish this in the radially symmetric case. In other words, we are only able to construct a function $\Psi$ for which we can estimate the right-hand side of (5.2) for radially symmetric functions $u$.

Before stating this result, we rewrite formula (5.2) for radially symmetric $\Psi$ and $u$. Consider a real-valued function $\Psi \in C^{\infty}\left(\mathbb{R}^{N}\right) \cap W^{4, \infty}\left(\mathbb{R}^{N}\right)$ as in Lemma 5.1, and assume further that $\Psi$ is radially symmetric. It follows that

so that

$$
\partial_{j k}^{2} \Psi=\frac{\delta_{j k}}{r} \Psi^{\prime}-\frac{x_{j} x_{k}}{r^{3}} \Psi^{\prime}+\frac{x_{j} x_{k}}{r^{2}} \Psi^{\prime \prime}
$$

$$
\begin{aligned}
\Re\langle H(\Psi) \nabla \bar{u}, \nabla u\rangle & =\frac{\Psi^{\prime}}{r}|\nabla u|^{2}-\left(\frac{\Psi^{\prime}}{r^{3}}-\frac{\Psi^{\prime \prime}}{r^{2}}\right)|x \cdot \nabla u|^{2} \\
& =\frac{\Psi^{\prime}}{r}|\nabla u|^{2}-\left(\frac{\Psi^{\prime}}{r}-\Psi^{\prime \prime}\right)\left|\partial_{r} u\right|^{2} .
\end{aligned}
$$

If, in addition, $u$ is radially symmetric, then (5.20) yields

$$
\Re\langle H(\Psi) \nabla \bar{u}, \nabla u\rangle=\Psi^{\prime \prime}\left|u_{r}\right|^{2} .
$$

It follows from (5.2) and (5.21) that if both $u$ and $\Psi$ are radially symmetric, then

$$
\begin{array}{r}
\frac{1}{2} \frac{d^{2}}{d t^{2}} \int_{\mathbb{R}^{N}} \Psi|u|^{2}=2 N \alpha E(u(t))-(N \alpha-4) \int_{\mathbb{R}^{N}}\left|u_{r}\right|^{2}-2 \int_{\mathbb{R}^{N}}\left(2-\Psi^{\prime \prime}\right)\left|u_{r}\right|^{2} \\
+\frac{\alpha}{\alpha+2} \int_{\mathbb{R}^{N}}(2 N-\Delta \Psi)|u|^{\alpha+2}-\frac{1}{2} \int_{\mathbb{R}^{N}} \Delta^{2} \Psi|u|^{2} \\
+\cos \theta \frac{d}{d t} \int_{\mathbb{R}^{N}}\left\{-2 \Psi|\nabla u|^{2}+\frac{\alpha+4}{\alpha+2} \Psi|u|^{\alpha+2}+\Delta \Psi|u|^{2}\right\} \\
-2 \cos ^{2} \theta \int_{\mathbb{R}^{N}} \Psi\left|u_{t}\right|^{2}
\end{array}
$$


Since $\Psi(x)$ is radially symmetric, by abuse of notation, we often write $\Psi(x)=\Psi(r)$, where $r=|x|$. Using this notation, we have $\Delta \Psi(x)=\Psi^{\prime \prime}(r)+\frac{N-1}{r} \Psi^{\prime}(r)$. We hope the reader will forgive our using both notations in the same formula, as we did in (5.22).

We now state the needed estimate. Since the proof is an adaptation of arguments in [18] and is somewhat technical, it is given in the appendix A to this paper.

Lemma 5.3. Suppose $N \geq 2$ and $\alpha \leq 4$. Given any $0<a, A<\infty$, there exists a radially symmetric function $\Psi \in C^{\infty}\left(\mathbb{R}^{N}\right) \cap W^{4, \infty}\left(\mathbb{R}^{N}\right)$, such that $\Psi(x)>0$ for $x \neq 0$ and

$$
-2 \int_{\mathbb{R}^{N}}\left(2-\Psi^{\prime \prime}\right)\left|u_{r}\right|^{2}+\frac{\alpha}{\alpha+2} \int_{\mathbb{R}^{N}}(2 N-\Delta \Psi)|u|^{\alpha+2}-\frac{1}{2} \int_{\mathbb{R}^{N}} \Delta^{2} \Psi|u|^{2} \leq a,
$$

for all radially symmetric $u \in H^{1}\left(\mathbb{R}^{N}\right)$ such that $\|u\|_{L^{2}} \leq A$.

Proof of Theorem 1.5. We let $K$ be defined by (5.10) and we set

$$
\tau^{\theta}=\sup \left\{t \in\left[0, T_{\max }^{\theta}\right) ;\left\|u^{\theta}(s)\right\|_{L^{2}}^{2} \leq K\left\|u_{0}\right\|_{L^{2}}^{2} \text { for } 0 \leq s \leq t\right\},
$$

so that

$$
\sup _{0 \leq \theta<\frac{\pi}{2}} \sup _{0 \leq t<\tau^{\theta}}\left\|u^{\theta}(t)\right\|_{L^{2}}^{2} \leq K\left\|u_{0}\right\|_{L^{2}}^{2} .
$$

It follows from Lemma 5.2 that

$$
T_{\max }^{\theta} \leq \frac{\alpha+4}{\alpha} \tau^{\theta} .
$$

We now let $\Psi$ be given by Lemma 5.3 with

$$
A=\sqrt{K}\left\|u_{0}\right\|_{L^{2}}, \quad a=-N \alpha E\left(u_{0}\right) .
$$

Since $E\left(u^{\theta}(t)\right) \leq E\left(u_{0}\right)$ it follows from (5.22), (5.23) and (5.27) that

$$
\begin{aligned}
& \frac{1}{2} \frac{d^{2}}{d t^{2}} \int_{\mathbb{R}^{N}} \Psi\left|u^{\theta}\right|^{2} \leq N \alpha E\left(u_{0}\right) \\
&+\cos \theta \frac{d}{d t} \int_{\mathbb{R}^{N}}\left\{-2 \Psi\left|\nabla u^{\theta}\right|^{2}+\frac{\alpha+4}{\alpha+2} \Psi\left|u^{\theta}\right|^{\alpha+2}+\Delta \Psi\left|u^{\theta}\right|^{2}\right\},
\end{aligned}
$$

for all $0 \leq t<\tau^{\theta}$. Let

$$
B=\int_{\mathbb{R}^{N}}\left\{-2 \Psi\left|\nabla u_{0}\right|^{2}+\frac{\alpha+4}{\alpha+2} \Psi\left|u_{0}\right|^{\alpha+2}+\Delta \Psi\left|u_{0}\right|^{2}\right\},
$$

and

$$
\begin{aligned}
\Gamma_{\theta}=\cos \theta\left(-\int_{\mathbb{R}^{N}} \Psi\left|\nabla u_{0}\right|^{2}+\int_{\mathbb{R}^{N}} \Psi\left|u_{0}\right|^{\alpha+2}+\frac{1}{2} \int_{\mathbb{R}^{N}} \Delta \Psi\left|u_{0}\right|^{2}\right) \\
+\sin \theta \Im \int_{\mathbb{R}^{N}} \nabla \Psi \overline{u_{0}} \nabla u_{0} .
\end{aligned}
$$

Integrating twice the inequality (5.28) and applying (5.29)-(5.30) and (5.1), we deduce that

$$
\begin{aligned}
& \frac{1}{2} \int_{\mathbb{R}^{N}} \Psi\left|u^{\theta}\right|^{2} \leq \frac{1}{2} \int_{\mathbb{R}^{N}} \Psi\left|u_{0}\right|^{2}+t \Gamma_{\theta}+N \alpha E\left(u_{0}\right) \frac{t^{2}}{2} \\
& \quad+\cos \theta \int_{0}^{t} \int_{\mathbb{R}^{N}}\left\{-2 \Psi\left|u_{r}^{\theta}\right|^{2}+\frac{\alpha+4}{\alpha+2} \Psi\left|u^{\theta}\right|^{\alpha+2}+\Delta \Psi\left|u^{\theta}\right|^{2}\right\}-B t \cos \theta .
\end{aligned}
$$


On the other hand, it follows from (2.8) that

$$
\frac{d}{d t} \int_{\mathbb{R}^{N}}\left|u^{\theta}\right|^{2} \geq 2 \cos \theta \frac{\alpha}{\alpha+2} \int_{\mathbb{R}^{N}}\left|u^{\theta}\right|^{\alpha+2} .
$$

Integrating between 0 and $t \in\left(0, \tau^{\theta}\right)$, we obtain

$$
2 \cos \theta \int_{0}^{t} \int_{\mathbb{R}^{N}}\left|u^{\theta}\right|^{\alpha+2} \leq \frac{\alpha+2}{\alpha}\left[\left\|u^{\theta}(t)\right\|_{L^{2}}^{2}-\left\|u_{0}\right\|_{L^{2}}^{2}\right] \leq \frac{\alpha+2}{\alpha}(K-1)\left\|u_{0}\right\|_{L^{2}}^{2}
$$

where we used (5.25) in the last inequality. Since $\Psi \in W^{4, \infty}\left(\mathbb{R}^{N}\right)$, it now follows from (5.31), (5.32) and (5.25) that there exists a constant $C$ independent of $\theta \in$ $(-\pi / 2, \pi / 2)$ and $t \in\left(0, \tau^{\theta}\right)$ such that

$$
0 \leq C+C t+N \alpha E\left(u_{0}\right) \frac{t^{2}}{2}
$$

for all $0 \leq t<\tau^{\theta}$. Since $E\left(u_{0}\right)<0$, this implies that there exists $T<\infty$ such that $\tau^{\theta} \leq T$ for all $-\frac{\pi}{2}<\theta<\frac{\pi}{2}$, and the result follows by applying (5.26).

Remark 5.4. Suppose $N \geq 2, \alpha<4 / N$. Let $u_{0} \in C_{0}\left(\mathbb{R}^{N}\right) \cap H^{1}\left(\mathbb{R}^{N}\right)$ be radially symmetric and satisfy $E\left(u_{0}\right)<0$. Given $-\pi / 2<\theta<\pi / 2$, let $u^{\theta}$ be the corresponding solution of (GL) defined on the maximal interval $\left[0, T_{\max }^{\theta}\right)$. It follows in particular from Theorem 1.1 that $u^{\theta}$ blows up in finite time. Using the calculations of the proof of Theorem 1.5, one can improve the estimate (1.4). More precisely, taking into account the term $(4-N \alpha) \int_{\mathbb{R}^{N}}\left|u_{r}^{\theta}\right|^{2}$ in (5.22), instead of (5.33), we obtain the inequality

$$
0 \leq C+C t+(4-N \alpha) \int_{0}^{t} \int_{0}^{s} \int_{\mathbb{R}^{N}}\left|u_{r}^{\theta}\right|^{2}+N \alpha E\left(u_{0}\right) \frac{t^{2}}{2}
$$

for all $-\pi / 2<\theta<\pi / 2$ and $0 \leq t<\tau^{\theta}$. On the other hand, it follows from (2.9) that

$$
\frac{d}{d t} \int_{\mathbb{R}^{N}}\left|u^{\theta}\right|^{2} \geq \alpha \cos \theta \int_{\mathbb{R}^{N}}\left|u_{r}^{\theta}\right|^{2} .
$$

Integrating between 0 and $t \in\left(0, \tau^{\theta}\right)$ and using (5.25), we obtain

$$
\alpha \cos \theta \int_{0}^{t} \int_{\mathbb{R}^{N}}\left|u_{r}^{\theta}\right|^{2} \leq\left[\left\|u^{\theta}(t)\right\|_{L^{2}}^{2}-\left\|u_{0}\right\|_{L^{2}}^{2}\right] \leq(K-1)\left\|u_{0}\right\|_{L^{2}}^{2} .
$$

It follows from (5.34) and (5.35) that for some constant $C>0$

$$
0 \leq C+C\left(1+\frac{4-N \alpha}{\cos \theta}\right) t+N \alpha E\left(u_{0}\right) \frac{t^{2}}{2},
$$

for all $-\pi / 2<\theta<\pi / 2$ and $0 \leq t<\tau^{\theta}$, which yields the estimate

$$
T_{\max }^{\theta} \leq C\left(u_{0}\right)\left(1+\frac{4-N \alpha}{\cos \theta}\right) .
$$

This is interesting, because we see the dependence in both $\theta$ and $\alpha$. It is optimal in $\theta$, but maybe not in $\alpha$. (Compare the lower estimate (4.12).) 


\section{Comments on the hypotheses of Theorem 1.5}

As observed above, the assumptions that $u_{0}$ is radially symmetric and that $\alpha \leq 4$ in Theorem 1.5 may seem unnatural. In this section, we show that both these assumptions are necessary for the method we use. Indeed, our proof of Theorem 1.5 relies on the identity (5.2). Assuming that $\Psi \in W^{4, \infty}\left(\mathbb{R}^{N}\right) \cap C^{4}\left(\mathbb{R}^{N}\right)$ is radially symmetric, it follows from (5.2) and (5.21) that

$$
\begin{aligned}
& \frac{1}{2} \frac{d^{2}}{d t^{2}} \int_{\mathbb{R}^{N}} \Psi|u|^{2}=2 N \alpha E(u(t)) \\
& -(N \alpha-4) \int_{\mathbb{R}^{N}}|\nabla u|^{2}+2 \int_{\mathbb{R}^{N}}\left(\frac{\Psi^{\prime}}{r}-\Psi^{\prime \prime}\right)\left(|\nabla u|^{2}-\left|u_{r}\right|^{2}\right)-2 \int_{\mathbb{R}^{N}}\left(2-\Psi^{\prime \prime}\right)|\nabla u|^{2} \\
& \quad+\frac{\alpha}{\alpha+2} \int_{\mathbb{R}^{N}}(2 N-\Delta \Psi)|u|^{\alpha+2}-\frac{1}{2} \int_{\mathbb{R}^{N}} \Delta^{2} \Psi|u|^{2} \\
& \quad+\cos \theta \frac{d}{d t} \int_{\mathbb{R}^{N}}\left\{-2 \Psi|\nabla u|^{2}+\frac{\alpha+4}{\alpha+2} \Psi|u|^{\alpha+2}+\Delta \Psi|u|^{2}\right\}-2 \cos ^{2} \theta \int_{\mathbb{R}^{N}} \Psi\left|u_{t}\right|^{2} .
\end{aligned}
$$

In order to complete our argument, we need at the very least an estimate of the form

$$
\begin{aligned}
& -(N \alpha-4) \int_{\mathbb{R}^{N}}|\nabla u|^{2}+2 \int_{\mathbb{R}^{N}}\left(\frac{\Psi^{\prime}}{r}-\Psi^{\prime \prime}\right)\left(|\nabla u|^{2}-\left|u_{r}\right|^{2}\right) \\
& \quad-2 \int_{\mathbb{R}^{N}}\left(2-\Psi^{\prime \prime}\right)|\nabla u|^{2}+\frac{\alpha}{\alpha+2} \int_{\mathbb{R}^{N}}(2 N-\Delta \Psi)|u|^{\alpha+2} \leq F\left(\|u\|_{L^{2}}\right),
\end{aligned}
$$

where $F$ is bounded on bounded sets. Lemma 5.3 provides such an estimate for radially symmetric $u$ under the assumption $\alpha \leq 4$.

We claim that if $N \alpha>4$, then there is no radially symmetric $\Psi \in C^{4}\left(\mathbb{R}^{N}\right) \cap$ $L^{\infty}\left(\mathbb{R}^{N}\right), \Psi \geq 0$, such that the estimate (6.1) holds for general $u$. To see this, fix $\varphi \in C_{\mathrm{c}}^{\infty}\left(\mathbb{R}^{N}\right), \varphi \not \equiv 0$ and let

$$
u(x)=\lambda^{N / 2} \varphi\left(\lambda\left(x-x_{0}\right)\right),
$$

where $\lambda>0$ and $x_{0} \in \mathbb{R}^{N}$. It follows in particular that $\|u\|_{L^{2}}=\|\varphi\|_{L^{2}}$. Given $g \in C\left(\mathbb{R}^{N}\right)$ we have for $\lambda$ large

$$
\begin{gathered}
\int_{\mathbb{R}^{N}} g(x)|\nabla u|^{2} \approx \lambda^{2} g\left(x_{0}\right) \int_{\mathbb{R}^{N}}|\nabla \varphi|^{2} d y, \\
\int_{\mathbb{R}^{N}} g(x)|u|^{\alpha+2} \approx \lambda^{N \alpha / 2} g\left(x_{0}\right) \int_{\mathbb{R}^{N}}|\varphi|^{\alpha+2} d y, \\
\int_{\mathbb{R}^{N}} g(x)\left|\partial_{r} u\right|^{2} \approx \lambda^{2} g\left(x_{0}\right) \int_{\mathbb{R}^{N}}\left|\partial_{r} \varphi\right|^{2} d y .
\end{gathered}
$$

If $N \alpha>4$ and (6.1) holds, then we deduce from (6.3)-(6.5) that $2 N-\Delta \Psi\left(x_{0}\right) \leq 0$ for all $x_{0} \in \mathbb{R}^{N}$, so that $\Psi \notin L^{\infty}\left(\mathbb{R}^{N}\right)$.

We now show that the assumption $\alpha \leq 4$ is necessary in order that (6.1) holds for some $\Psi \in W^{4, \infty}\left(\mathbb{R}^{N}\right) \cap C^{4}\left(\mathbb{R}^{N}\right)$ and all radially symmetric $u$. To see this, fix $\varphi \in C^{\infty}\left([0, \infty)\right.$ with $\operatorname{supp} \varphi \subset[1,2]$ and $\varphi \not \equiv 0$. For $\lambda>0$ and $r_{0}>0$ consider

$$
u(x)=\lambda^{1 / 2} r_{0}^{-(N-1) / 2} \varphi\left(\lambda\left(r-r_{0}\right)\right) .
$$


Denote by $\omega_{N}$ the area of the unitary sphere of $\mathbb{R}^{N}$. It follows that for $\lambda \geq 2 / r_{0}$,

$$
\begin{aligned}
\|u\|_{L^{2}}^{2} & =\omega_{N} \lambda r_{0}^{-N+1} \int_{0}^{\infty}\left|\varphi\left(\lambda\left(r-r_{0}\right)\right)\right|^{2} r^{N-1} d r \\
& =\omega_{N}\left(\lambda r_{0}\right)^{-N+1} \int_{1}^{2}|\varphi(r)|^{2}\left(r+\lambda r_{0}\right)^{N-1} d r \\
& \leq \omega_{N}\left(\lambda r_{0}\right)^{-N+1}\left(2+\lambda r_{0}\right)^{N-1}\|\varphi\|_{L^{2}(\mathbb{R})}^{2} \leq 2^{N-1} \omega_{N}\|\varphi\|_{L^{2}(\mathbb{R})}^{2} .
\end{aligned}
$$

Given a radially symmetric function $g \in C\left(\mathbb{R}^{N}\right)$ and $r_{0}>0$ such that $g\left(r_{0}\right)>0$, we have as $\lambda \rightarrow \infty$

$$
\begin{aligned}
\int_{\mathbb{R}^{N}} g(x)\left|u_{r}\right|^{2} & =\omega_{N} \lambda^{2}\left(\lambda r_{0}\right)^{-N+1} \int_{1}^{2} g\left(\lambda^{-1} r+r_{0}\right) \mid \varphi^{\prime}(r) \|^{2}\left(r+\lambda r_{0}\right)^{N-1} d r \\
& \approx \lambda^{2} \omega_{N} g\left(r_{0}\right)\left\|\varphi^{\prime}\right\|_{L^{2}(\mathbb{R})}^{2},
\end{aligned}
$$

and, similarly,

$$
\begin{array}{r}
\int_{\mathbb{R}^{N}} g(x)|u|^{\alpha+2}=\omega_{N} \lambda^{\frac{\alpha}{2}} r_{0}^{-\frac{(N-1)(\alpha+2)}{2}} \int_{1}^{2} g\left(\lambda^{-1} r+r_{0}\right)|\varphi(r)|^{\alpha+2}\left(\lambda^{-1} r+r_{0}\right)^{N-1} d r \\
\approx \lambda^{\frac{\alpha}{2}} \omega_{N} g\left(r_{0}\right) r_{0}^{-\frac{(N-1) \alpha}{2}}\|\varphi\|_{L^{\alpha+2}(\mathbb{R})}^{\alpha+2} . \quad(6.9)
\end{array}
$$

If $\alpha>4$ and (6.1) holds, then we deduce from (6.7)-(6.9) that $2 N-\Delta \Psi\left(r_{0}\right) \leq 0$ for all $r_{0}>0$, so that $\Psi \notin L^{\infty}\left(\mathbb{R}^{N}\right)$.

\section{The VARIANCE IDENTITy AND CONSEqUenCes}

Another way one might try to dispense with the requirements in Theorem 1.5 that $\alpha \leq 4$ and that $u_{0}$ be radially symmetric is to assume that $u_{0}$ has finite variance. Indeed, finite time blowup of negative energy solutions of the nonlinear Schrödinger equation, i.e. (GL) with $\theta= \pm \pi / 2$, was originally proved [9,32] for finite variance solutions. No assumption of radial symmetry nor the upper bound $\alpha \leq 4$ was required. These conditions were introduced by Ogawa and Tsutsumi [18] in their proof of finite time blowup of negative energy solutions (with possibly infinite variance). Therefore, it is reasonable to hope that for (GL) the additional assumption of finite variance could lead to a proof of finite time blowup without the assumptions in [18].

Consequently, we consider a finite variance solution of (GL) which is sufficiently regular so that $\Psi=|x|^{2}$ can be used in formula (5.2). This gives

$$
\begin{aligned}
\frac{1}{2} \frac{d^{2}}{d t^{2}} \int_{\mathbb{R}^{N}}|x|^{2}|u|^{2} & =2 N \alpha E(u(t))-(N \alpha-4) \int_{\mathbb{R}^{N}}|\nabla u|^{2} \\
+\cos \theta \frac{d}{d t} \int_{\mathbb{R}^{N}}\left\{-2|x|^{2}|\nabla u|^{2}+\frac{\alpha+4}{\alpha+2}|x|^{2}|u|^{\alpha+2}+2 N|u|^{2}\right\} & -2 \cos ^{2} \theta \int_{\mathbb{R}^{N}}|x|^{2}\left|u_{t}\right|^{2} .
\end{aligned}
$$

These formal calculations can be justified by standard techniques assuming $u_{0}$ is sufficiently regular, and certainly if $u_{0} \in C_{\mathrm{c}}^{\infty}\left(\mathbb{R}^{N}\right)$. We note right away that the three terms estimated in Lemma 5.3 have disappeared, and so this lemma is no longer needed. We therefore proceed to outline a proof of the conclusion of Theorem 1.5 based on the formula (7.1). Unfortunately, it will turn out that the conditions that 
$\alpha \leq 4$ and that $u_{0}$ be radially symmetric will again be required, but for apparently different reasons than in the proof of Lemma 5.3.

Consider, for simplicity, an initial value $u_{0} \in C_{\mathrm{c}}^{\infty}\left(\mathbb{R}^{N}\right)$. Suppose (1.3) and let $u^{\theta}$ be the corresponding solution of (GL), defined on the maximal interval $\left[0, T_{\max }^{\theta}\right)$.

Arguing as in the proof of Theorem 1.5 at the end of Section 5, we obtain that for some $C_{1}>0$ independent of $\theta$

$$
\begin{aligned}
\int_{\mathbb{R}^{N}}|x|^{2}\left|u^{\theta}\right|^{2} & \leq \int_{\mathbb{R}^{N}}|x|^{2}\left|u_{0}\right|^{2}+C_{1} t+N \alpha E\left(u_{0}\right) t^{2} \\
& +2 \cos \theta \int_{0}^{t} \int_{\mathbb{R}^{N}}\left\{-2|x|^{2}\left|\nabla u^{\theta}\right|^{2}+\frac{\alpha+4}{\alpha+2}|x|^{2}\left|u^{\theta}\right|^{\alpha+2}+2 N\left|u^{\theta}\right|^{2}\right\},
\end{aligned}
$$

for all $0 \leq t<T_{\max }^{\theta}$, see (5.30), (5.29) and (5.31). For $K$ defined by (5.10) set $C_{2}=4 N K\left\|u_{0}\right\|_{L^{2}}^{2}$. If $\tau^{\theta}$ is given by (5.24) then

$$
4 N \cos \theta \int_{0}^{t} \int_{\mathbb{R}^{N}}\left|u^{\theta}\right|^{2} \leq C_{2} t
$$

for all $0 \leq t<\tau^{\theta}$, see (5.25). Therefore, in order to obtain an inequality analogous to $(5.33)$ it remains to estimate the term

$$
2 \cos \theta \int_{0}^{t} \int_{\mathbb{R}^{N}}\left\{-2|x|^{2}\left|\nabla u^{\theta}\right|^{2}+\frac{\alpha+4}{\alpha+2}|x|^{2}\left|u^{\theta}\right|^{\alpha+2}\right\} .
$$

This can be done with the following estimate, similar to some results in [2].

Lemma 7.1. Suppose $N \geq 2$ and $4 / N \leq \alpha \leq 4$. Given any $M>0$, there exists a constant $C$ such that

$$
\int|x|^{2}|u|^{\alpha+2} \leq \int|x|^{2}|\nabla u|^{2}+C \int|u|^{\alpha+2}+C,
$$

for all smooth, radially symmetric $u$ such that $\|u\|_{L^{2}} \leq M$.

Proof. We first claim that

$$
\left\||\cdot|{ }^{N}|u|^{2}\right\|_{L^{\infty}} \leq 2\|u\|_{L^{2}}\||\cdot| \nabla u\|_{L^{2}}
$$

Indeed, considering $u$ as a function of $r>0$, we have

$$
r^{N}|u(r)|^{2}=-\int_{r}^{\infty} \frac{d}{d s}\left[s^{N}|u(s)|^{2}\right]=-N \int_{r}^{\infty} s^{N-1}|u(s)|^{2}+2 \int_{r}^{\infty} s^{N} \Re\left(\bar{u} \partial_{r} u\right) .
$$

We deduce that

$$
\begin{aligned}
r^{N}|u(r)|^{2} & \leq 2 \int_{r}^{\infty} s^{N}|u(s)|\left|\partial_{r} u(s)\right| \\
& \leq 2\left(\int_{r}^{\infty} s^{N-1}|u(s)|^{2}\right)^{\frac{1}{2}}\left(\int_{r}^{\infty} s^{N+1}\left|\partial_{r} u(s)\right|^{2}\right)^{\frac{1}{2}} \\
& =2\|u\|_{L^{2}(\{|x|>r\})}\||\cdot| \nabla u\|_{L^{2}(\{|x|>r\}),}
\end{aligned}
$$

which proves (7.6). It now follows from (7.6) that

$$
\int|x|^{2}|u|^{\alpha+2} \leq\left\||\cdot|{ }^{N}|u|^{2}\right\|_{L^{\infty}}^{\frac{2}{N}} \int|u|^{\alpha+2-\frac{4}{N}} \leq 2^{\frac{2}{N}} M^{\frac{2}{N}}\||\cdot| \nabla u\|_{L^{2}}^{\frac{2}{N}} \int|u|^{\alpha+2-\frac{4}{N}} .
$$

Since, by Hölder,

$$
\int|u|^{\alpha+2-\frac{4}{N}} \leq M^{\frac{8}{N \alpha}}\left(\int|u|^{\alpha+2}\right)^{\frac{N \alpha-4}{N \alpha}}
$$


we deduce that

$$
\int|x|^{2}|u|^{\alpha+2} \leq 2^{\frac{2}{N}} M^{\frac{2 \alpha+8}{N \alpha}}\left(\int|u|^{\alpha+2}\right)^{\frac{N \alpha-4}{N \alpha}}\||\cdot| \nabla u\|_{L^{2}}^{\frac{2}{N}} .
$$

Suppose first that $\alpha>4 / N$ and fix $0<\eta \leq 1$. Applying Young's inequality $x y \leq \eta^{-\frac{p}{p^{\prime}}} \frac{x^{p}}{p}+\eta \frac{y^{p^{\prime}}}{p^{\prime}}$ with $\frac{1}{p}=\frac{N \alpha-4}{N \alpha}$, it follows that

$$
2^{-\frac{2}{N}} \int|x|^{2}|u|^{\alpha+2} \leq \eta^{-\frac{4}{N \alpha-4}} \frac{N \alpha-4}{N \alpha} \int|u|^{\alpha+2}+\eta \frac{4}{N \alpha} M^{\frac{\alpha+4}{2}}\||\cdot| \nabla u\|_{L^{2}}^{\frac{\alpha}{2}} .
$$

If $\alpha<4$, then we apply again Young's inequality to the last term in the right-hand side of (7.8) and we obtain

$$
\begin{aligned}
2^{-\frac{2}{N}} \int|x|^{2}|u|^{\alpha+2} \leq \eta^{-\frac{4}{N \alpha-4}} \frac{N \alpha-4}{N \alpha} \int|u|^{\alpha+2}+\frac{\eta}{N}\||\cdot| \nabla u\|_{L^{2}}^{2} & \\
& +\frac{\eta(4-\alpha)}{N \alpha} M^{\frac{2 \alpha+8}{4-\alpha}} .
\end{aligned}
$$

The estimate (7.5) follows by choosing appropriately $\eta$. If $\alpha=4$ (note that $4>4 / N$ since $N>1$ ), then (7.5) follows from (7.8) by choosing $\eta$ sufficiently small. It remains to consider the case $\alpha=4 / N$, in which (7.7) becomes

$$
\int|x|^{2}|u|^{\alpha+2} \leq 2^{\frac{2}{N}} M^{\frac{2 \alpha+8}{N \alpha}}\||\cdot| \nabla u\|_{L^{2}}^{\frac{2}{N}} .
$$

Since $N>1$, we may apply Young's inequality to deduce (7.5).

Assuming $N \geq 2,4 / N \leq \alpha \leq 4$ and $u_{0}$ is radially symmetric, one can then continue as follows. Setting $M=\sqrt{K}\left\|u_{0}\right\|_{L^{2}}$, we deduce from (5.25) and Lemma 7.1 that there exists a constant $C_{3}>0$ such that

$$
\int_{\mathbb{R}^{N}}\left\{-2|x|^{2}\left|\nabla u^{\theta}\right|^{2}+\frac{\alpha+4}{\alpha+2}|x|^{2}\left|u^{\theta}\right|^{\alpha+2}\right\} \leq C_{3}+C_{3} \int_{\mathbb{R}^{N}}\left|u^{\theta}\right|^{\alpha+2},
$$

for all $0 \leq \theta<\frac{\pi}{2}$ and all $0 \leq t<\tau^{\theta}$. It follows from (7.2), (7.3) and (7.10) that

$$
\begin{aligned}
\int_{\mathbb{R}^{N}}|x|^{2}\left|u^{\theta}\right|^{2} \leq \int_{\mathbb{R}^{N}}|x|^{2}\left|u_{0}\right|^{2}+\left(C_{1}+C_{2}+2 C_{3}\right) t & \left.+N \alpha E\left(u_{0}\right)\right) t^{2} \\
& +2 C_{3} \cos \theta \int_{0}^{t} \int_{\mathbb{R}^{N}}\left|u^{\theta}\right|^{\alpha+2} .
\end{aligned}
$$

Using (5.32) we see that there exists $C_{4}$ such that

$$
\int_{\mathbb{R}^{N}}|x|^{2}\left|u^{\theta}\right|^{2} \leq C_{4}+\left(C_{1}+C_{2}+2 C_{3}\right) t+N \alpha E\left(u_{0}\right) t^{2}
$$

for all $-\frac{\pi}{2} \leq \theta<\frac{\pi}{2}$ and all $0 \leq t<\tau^{\theta}$. We then may conclude as in the proof of Theorem 1.5.

Thus we see how to obtain a uniform estimate of $T_{\max }^{\theta}$ by using the variance identity. However, we use Lemma 7.1 and this is why we assume that $u_{0}$ is radially symmetric and that $N \geq 2$ and $4 / N \leq \alpha \leq 4$. Therefore, we obtain a weaker result than Theorem 1.5 (which does not require finite variance).

The obstacle for improving this argument seems to be Lemma 7.1. Unfortunately, both the symmetry assumption and the requirement $\alpha \leq 4$ are necessary in Lemma 7.1. 
Let us first observe that radial symmetry is essential in Lemma 7.1. Indeed, fix $\varphi \in C_{\mathrm{c}}^{\infty}\left(\mathbb{R}^{N}\right), \varphi \not \equiv 0$ and let $u(x)$ be given by (6.2). Taking $g(x) \equiv|x|^{2}$ in (6.3) and (6.4) and $g(x) \equiv 1$ in (6.4), we see that (7.5) cannot hold for arbitrarly large $\left|x_{0}\right|$ when $N \alpha>4$. (And not even for $N \alpha=4$, since we may choose $\varphi$ such that $\|\varphi\|_{L^{\alpha+2}}^{\alpha+2} \gg\|\nabla \varphi\|_{L^{2}}^{2}$.)

We next remark that the restriction $\alpha \leq 4$ is also essential in Lemma 7.1. Indeed, let $u$ be defined by (6.6) for some $\varphi \in \bar{C}^{\infty}(\mathbb{R}), \varphi \neq \equiv 0$ supported in $[1,2]$ and for $\lambda, r_{0}>0$. Applying the first identity in (6.8) with $g(x) \equiv|x|^{2}$ and the first identity in (6.9) with $g(x) \equiv 1$, we deduce that

$$
\begin{gathered}
\int|x|^{2}|\nabla u|^{2} \leq \lambda^{2} 2^{N+1} \omega_{N} r_{0}^{2}\left\|\varphi^{\prime}\right\|_{L^{2}(\mathbb{R})}^{2}, \\
\int|u|^{\alpha+2} \leq \lambda^{\frac{\alpha}{2}} 2^{N-1} \omega_{N} r_{0}^{-\frac{(N-1) \alpha}{2}}\|\varphi\|_{L^{\alpha+2}(\mathbb{R})}^{\alpha+2},
\end{gathered}
$$

for all $\lambda \geq 2 / r_{0}$. Moreover, applying the first identity in (6.9) with $g(x) \equiv|x|^{2}$, we obtain

$$
\int|x|^{2}|u|^{\alpha+2} \geq \lambda^{\frac{\alpha}{2}} \omega_{N} r_{0}^{2-\frac{(N-1) \alpha}{2}}\|\varphi\|_{L^{\alpha+2}(\mathbb{R})}^{\alpha+2},
$$

for all $\lambda>0$. Applying (6.7) and (7.12)-(7.14), we see that if (7.5) holds then there is a constant $A>0$ such that

$$
\lambda^{\frac{\alpha}{2}} r_{0}^{2-\frac{(N-1) \alpha}{2}} \leq A\left(1+\lambda^{2} r_{0}^{2}+\lambda^{\frac{\alpha}{2}} r_{0}^{-\frac{(N-1) \alpha}{2}}\right)
$$

for all $r_{0}>0$ and $\lambda \geq 2 / r_{0}$. Taking $r_{0}=\sqrt{2 A}$, we obtain

$$
\lambda^{\frac{\alpha}{2}} r_{0}^{-\frac{(N-1) \alpha}{2}} \leq 1+\lambda^{2} r_{0}^{2}
$$

for all $\lambda \geq 2 / r_{0}$, which yields $\alpha \leq 4$.

\section{Appendix A. Proof of Lemma 5.3}

We follow the method of [18], and we construct a family $\left(\Psi_{\varepsilon}\right)_{\varepsilon>0}$ such that, given $a, A$, the estimate (5.23) holds with $\Psi=\Psi_{\varepsilon}$ provided $\varepsilon>0$ is sufficiently small. Fix a function $h \in C^{\infty}([0, \infty))$ such that

$$
h \geq 0, \quad \operatorname{supp} h \subset[1,2], \quad \int_{0}^{\infty} h(s) d s=1,
$$

and let

$$
\zeta(t)=t-\int_{0}^{t}(t-s) h(s) d s=t-\int_{0}^{t} \int_{0}^{s} h(\sigma) d \sigma d s,
$$

for $t \geq 0$. It follows that $\zeta \in C^{\infty}([0, \infty)) \cap W^{4, \infty}\left((0, \infty), \zeta^{\prime} \geq 0, \zeta^{\prime \prime} \leq 0, \zeta(t)=t\right.$ for $t \leq 1$ and $\zeta(t)=M$ for $t \geq 2$ with $M=\int_{0}^{2} \operatorname{sh}(s) d s$. Set

$$
\Phi(x)=\zeta\left(|x|^{2}\right) .
$$

It follows in particular that $\Phi \in C^{\infty}\left(\mathbb{R}^{N}\right) \cap W^{4, \infty}\left(\mathbb{R}^{N}\right)$. Given any $\varepsilon>0$, set

$$
\Psi_{\varepsilon}(x)=\varepsilon^{-2} \Phi(\varepsilon x),
$$

so that

$$
\left\|\Delta^{2} \Psi_{\varepsilon}\right\|_{L^{\infty}}=\varepsilon^{2}\left\|\Delta^{2} \Phi\right\|_{L^{\infty}} .
$$


Next, set

$$
\xi(t)=\sqrt{2\left(1-\zeta^{\prime}(t)\right)-4 t \zeta^{\prime \prime}(t)}=\sqrt{2 \int_{0}^{t} h(s) d s+4 t h(t) .}
$$

It is not difficult to check that $\xi \in C^{1}([0, \infty)) \cap W^{1, \infty}(0, \infty)$. Let

$$
\gamma(r)=\xi\left(r^{2}\right),
$$

and, given $\varepsilon>0$, let

$$
\gamma_{\varepsilon}(r)=\gamma(\varepsilon r) \text {. }
$$

It easily follows that $\gamma_{\varepsilon}$ is supported in $\left[\varepsilon^{-1}, \infty\right)$, so that

$$
\left\|r^{-(N-1)} \gamma_{\varepsilon}^{\prime}\right\|_{L^{\infty}} \leq \varepsilon^{N-1}\left\|\gamma_{\varepsilon}^{\prime}\right\|_{L^{\infty}}=\varepsilon^{N}\left\|\gamma^{\prime}\right\|_{L^{\infty}}
$$

and

$$
\left\|r^{-(N-1)} \gamma_{\varepsilon} u_{r}\right\|_{L^{2}} \leq \varepsilon^{N-1}\left\|\gamma_{\varepsilon} u_{r}\right\|_{L^{2}}
$$

Set

$$
\begin{aligned}
I_{\varepsilon}(u)=-2 \int_{\mathbb{R}^{N}}(2- & \left.\Psi_{\varepsilon}^{\prime \prime}\right)\left|u_{r}\right|^{2} \\
& +\frac{\alpha}{\alpha+2} \int_{\mathbb{R}^{N}}\left(2 N-\Delta \Psi_{\varepsilon}\right)|u|^{\alpha+2}-\frac{1}{2} \int_{\mathbb{R}^{N}} \Delta^{2} \Psi_{\varepsilon}|u|^{2} .
\end{aligned}
$$

Elementary but long calculations using in particular (A.6) show that

$$
2-\Psi_{\varepsilon}^{\prime \prime}(x)=\gamma_{\varepsilon}(|x|)^{2},
$$

and

$$
2 N-\Delta \Psi_{\varepsilon}(x)=N\left[\gamma_{\varepsilon}(|x|)\right]^{2}+4(N-1)(\varepsilon|x|)^{2} \zeta^{\prime \prime}\left(\varepsilon^{2}|x|^{2}\right) \leq N\left[\gamma_{\varepsilon}(|x|)\right]^{2} .
$$

We deduce from (A.11), (A.12), (A.13) and (A.5) that

$$
I_{\varepsilon}(u) \leq-2 \int_{\mathbb{R}^{N}} \gamma_{\varepsilon}^{2}\left|u_{r}\right|^{2}+\frac{N \alpha}{\alpha+2} \int_{\mathbb{R}^{N}} \gamma_{\varepsilon}^{2}|u|^{\alpha+2}+\frac{\varepsilon^{2}}{2}\left\|\Delta^{2} \Phi\right\|_{L^{\infty}}\|u\|_{L^{2}}^{2} .
$$

We next claim that

$$
\left\|\gamma_{\varepsilon}^{\frac{1}{2}} u\right\|_{L^{\infty}}^{2} \leq \varepsilon^{N}\left\|\gamma^{\prime}\right\|_{L^{\infty}}\|u\|_{L^{2}}^{2}+2 \varepsilon^{N-1}\|u\|_{L^{2}}\left\|\gamma_{\varepsilon} u_{r}\right\|_{L^{2}}
$$

Indeed,

$$
\begin{aligned}
\gamma_{\varepsilon}(r)|u(r)|^{2} & =-\int_{r}^{\infty} \frac{d}{d s}\left[\gamma_{\varepsilon}(s)|u(s)|^{2}\right] \leq \int_{0}^{\infty}\left|\gamma_{\varepsilon}^{\prime}\right||u|^{2}+2 \int_{0}^{\infty} \gamma_{\varepsilon}|u|\left|u_{r}\right| \\
& \leq\left\|r^{-(N-1)} \gamma_{\varepsilon}^{\prime}\right\|_{L^{\infty}}\|u\|_{L^{2}}^{2}+2\|u\|_{L^{2}}\left\|r^{-(N-1)} \gamma_{\varepsilon} u_{r}\right\|_{L^{2}} .
\end{aligned}
$$

(The above calculation is valid for a smooth function $u$ and is easily justified for a general $u$ by density.) The estimate (A.15) follows from (A.16), (A.9) and (A.10). We now observe that

$$
\int_{\mathbb{R}^{N}} \gamma_{\varepsilon}^{2}|u|^{\alpha+2}=\int_{\mathbb{R}^{N}} \gamma_{\varepsilon}^{\frac{4-\alpha}{2}}\left[\gamma_{\varepsilon}^{\frac{1}{2}}|u|\right]^{\alpha}|u|^{2} \leq\|\gamma\|_{L^{\infty}}^{\frac{4-\alpha}{2}}\left\|\gamma_{\varepsilon}^{\frac{1}{2}} u\right\|_{L^{\infty}}^{\alpha}\|u\|_{L^{2}}^{2} .
$$

Applying (A.15) and the inequality $x^{\frac{\alpha}{2}} \leq 1+x^{2}$, we deduce from (A.17) that there exists a constant $C$ independent of $\varepsilon>0$ and $u$ such that

$$
\frac{N \alpha}{\alpha+2} \int_{\mathbb{R}^{N}} \gamma_{\varepsilon}^{2}|u|^{\alpha+2} \leq C \varepsilon^{\frac{(N-1) \alpha}{2}}\|u\|_{L^{2}}^{\frac{\alpha}{2}+2}\left(\varepsilon^{\frac{\alpha}{2}}\|u\|_{L^{2}}^{\frac{\alpha}{2}}+1+\left\|\gamma_{\varepsilon} u_{r}\right\|_{L^{2}}^{2}\right) .
$$


Estimates (A.14) and (A.18) now yield

$$
\begin{aligned}
I_{\varepsilon}(u) \leq-(2- & \left.C \varepsilon^{\frac{(N-1) \alpha}{2}}\|u\|_{L^{2}}^{\frac{\alpha}{2}+2}\right) \int_{\mathbb{R}^{N}} \gamma_{\varepsilon}^{2}\left|u_{r}\right|^{2} \\
& +C \varepsilon^{\frac{(N-1) \alpha}{2}}\|u\|_{L^{2}}^{\frac{\alpha}{2}+2}\left(\varepsilon^{\frac{\alpha}{2}}\|u\|_{L^{2}}^{\frac{\alpha}{2}}+1\right)+\frac{\varepsilon^{2}}{2}\left\|\Delta^{2} \Phi\right\|_{L^{\infty}}\|u\|_{L^{2}}^{2} .
\end{aligned}
$$

We now fix $0 \leq a, A<\infty$ and we first choose $\varepsilon>0$ sufficiently small so that $C \varepsilon^{\frac{(N-1) \alpha}{2}} A^{\frac{\alpha}{2}+2} \leq 2$. It then follows from (A.19) that if $\|u\|_{L^{2}} \leq A$, then

$$
I_{\varepsilon}(u) \leq C \varepsilon^{\frac{(N-1) \alpha}{2}} A^{\frac{\alpha}{2}+2}\left(\varepsilon^{\frac{\alpha}{2}} A^{\frac{\alpha}{2}}+1\right)+\frac{\varepsilon^{2}}{2}\left\|\Delta^{2} \Phi\right\|_{L^{\infty}} A^{2} .
$$

Choosing $\varepsilon>0$ possibly smaller, but depending on $a$, A, we deduce that $I_{\varepsilon}(u) \leq a$ if $\|u\|_{L^{2}} \leq A$. This completes the proof.

\section{REFERENCES}

[1] Ball J.M. Remarks on blow-up and nonexistence theorems for nonlinear evolution equations, Quart. J. Math. Oxford Ser. (2) 28 (1977), no. 112, 473-486. (MR0473484) (doi: $10.1093 /$ qmath/28.4.473)

[2] Caffarelli L., Kohn R. V. and Nirenberg L. First order interpolation inequalities with weights, Compositio Math. 53 (1984), no. 3, 259-275. (MR0768824) (link: http://www.numdam.org/item?id=CM_1984__53_3_259_0

[3] Doering C.R., Gibbon J.D., Holm, D.D. and Nicolaenko B. Low-dimensional behaviour in the complex Ginzburg-Landau equation. Nonlinearity 1 (1988), no. 2, 279-309. (MR0937004) (doi: 10.1088/0951-7715/1/2/001)

[4] Doering C.R., Gibbon J.D. and Levermore C.D. Weak and strong solutions of the complex Ginzburg-Landau equation, Phys. D 71 (1994), 285-318. (MR1264120) (doi: 10.1016/0167-2789(94)90150-3)

[5] Fife P.C.Mathematical aspects of reacting and diffusing systems, Lecture Notes in Biomathematics 28, Springer, New York, 1979. (MR0527914)

[6] Ginibre J. and Velo G. On a class of nonlinear Schrödinger equations. I. The Cauchy problem, general case, J. Funct. Anal. 32, no. 1 (1979), 1-32. (MR0533218) (doi: 10.1016/0022-1236(79)90076-4)

[7] Ginibre J. and Velo G. The Cauchy problem in local spaces for the complex Ginzburg Landau equation I: compactness methods, Phys. D 95 (1996), no. 3-4, 191-228. (MR1406282) (doi: 10.1016/0167-2789(96)00055-3)

[8] Ginibre J. and Velo G. The Cauchy problem in local spaces for the complex Ginzburg Landau equation II: contraction methods, Comm. Math. Phys. 187 (1997), no. 1, 45-79. (MR1463822) (doi: 10.1007/s002200050129)

[9] Glassey R.T. On the blowing up of solutions to the Cauchy problem for nonlinear Schrödinger equations, J. Math. Phys. 18 (1977), no. 9, 1794-1797. (MR0460850) (doi: 10.1063/1.523491)

[10] Haraux A. and Weissler F. B. Non-uniqueness for a semilinear initial value problem, Indiana Univ. Math. J. 31 (1982), no. 2, 167-189. (link: http://www.iumj.indiana.edu/IUMJ/FTDLOAD/1982/31/31016/pdf)

[11] Kato T. On nonlinear Schrödinger equations, Ann. Inst. H. Poincaré Phys. Théor. 46 (1987), no. 1, 113-129. (MR0877998) (link: http://www.numdam.org/item?id=AIHPA_1987_-46_1_113_0)

[12] Kavian O. A remark on the blowing-up of solutions to the Cauchy problem for nonlinear Schrödinger equations, Trans. Amer. Math. Soc. 299 (1987), no. 1, 193-205. (MR0869407) (link: http://www.jstor.org/stable/2000489)

[13] Levine H.A. Some nonexistence and instability theorems for formally parabolic equations of the form $P u_{t}=-A u+f(u)$, Arch. Ration. Mech. Anal. 51 (1973), 371-386. (MR0348216) (doi: 10.1007/BF00263041)

[14] Masmoudi N. and Zaag H. Blow-up profile for the complex Ginzburg-Landau equation, J. Funct. Anal. 255 (2008), no. 7, 1613-1666. (MR2442077) (doi: 10.1016/j.jfa.2008.03.008) 
[15] Mischaikow K. and Morita Y. Dynamics on the global attractor of a gradient flow arising from the Ginzburg-Landau equation. Japan J. Indust. Appl. Math. 11 (1994), no. 2, 185-202. (MR1286431) (doi: 10.1007/BF03167221)

[16] Nasibov Sh. M. On some sufficient conditions for the blow-up of solutions of the nonlinear Ginzburg-Landau-Schrödinger evolution equation, J. Appl. Math. 2004, no. 1, 23-35. (MR2077878) (doi: 10.1155/S1110757X04303049)

[17] Nasibov Sh. M. On the blow-up of solutions of a mixed problem for the nonlinear Ginzburg-Landau-Schrödinger evolution equation, Differ. Equ. 39 (2003), no. 8, 1144-1149. (MR2198240) (doi: 10.1023/B:DIEQ.0000011288.63604.b0)

[18] Ogawa T. and Tsutsumi Y. Blow-up of $H^{1}$ solutions for the nonlinear Schrödinger equation, J. Differential Equations 92 (1991), 317-330. (MR1120908) (doi: 10.1016/0022-0396(91)90052-B)

[19] Ogawa T. and Tsutsumi Y. Blow-up of $H^{1}$ solutions for the one dimensional nonlinear Schrödinger equation with critical power nonlinearity, Proc. Amer. Math. Soc. 111 (1991), 487-496. (MR1045145) (link: http://www.jstor.org/stable/2048340)

[20] Okazawa N. and Yokota T. Monotonicity method for the complex Ginzburg-Landau equation, including smoothing effect. Proceedings of the Third World Congress of Nonlinear Analysts, Part 1 (Catania, 2000). Nonlinear Anal. 47 (2001), no. 1, 79-88. (MR1970632) (doi: 10.1016/S0362-546X(01)00158-4)

[21] Okazawa N. and Yokota T. Global existence and smoothing effect for the complex Ginzburg-Landau equation with p-Laplacian, J. Differential Equations 182 (2002), 541-576. (MR1900334) (doi: 10.1006/jdeq.2001.4097)

[22] Okazawa N. and Yokota T. Monotonicity method applied to the complex GinzburgLandau and related equations, J. Math. Anal. Appl. 267 (2002), 247-263. (MR1886827) (doi: $10.1006 /$ jmaa.2001.7770)

[23] Okazawa N. and Yokota T. Perturbation theory for $m$-accretive operators and generalized complex Ginzburg-Landau equations, J. Math. Soc. Japan 54 (2002), 1-19. (MR1864925) (doi: $10.2969 / \mathrm{jmsj} / 1191593952$ )

[24] Ozawa T. and Yamazaki Y. Life-span of smooth solutions to the complex GinzburgLandau type equation on a torus, Nonlinearity 16 (2003), 2029-2034. (MR2012854) (doi: 10.1088/0951-7715/16/6/309)

[25] Plecháč P. and Šverák V. On self-similar singular solutions of the complex GinzburgLandau equation, Comm. Pure Appl. Math. 54 (2001), no. 10, 1215-1242. (MR1843986) (doi: 10.1002/cpa.3006)

[26] Popp S, Stiller O, Kuznetsov E. and Kramer L. The cubic complex GinzburgLandau equation for a backward bifurcation. Phys. D 114 (1998), no. 1-2, 81-107. (MR1612047)(doi: 10.1016/S0167-2789(97)00170-X)

[27] Rottschäfer V. Multi-bump, self-similar, blowup solutions of the Ginzburg-Landau equation. Phys. D 237 (2008), no. 4, 510-539. (MR2451604) (doi: 10.1016/j.physd.2007.09.022)

[28] Snoussi S and Tayachi S. Nonglobal existence of solutions for a generalized Ginzburg-Landau equation coupled with a Poisson equation, J. Math. Anal. Appl. 254 (2001), 558-570. (MR1805524) (doi: 10.1006/jmaa.2000.7235)

[29] Sulem C. and Sulem P.-L.The nonlinear Schrödinger equation. Self-focusing and wave collapse, Applied Mathematical Sciences. 139, Springer-Verlag, New York, 1999. (MR1696311)

[30] Weissler F.B. Local existence and nonexistence for semilinear parabolic equations in $L^{p}$, Indiana Univ. Math. J. 29 (1980), no. 1, 79-102. (MR0554819) (link: http://www.iumj.indiana.edu/IUMJ/FTDLOAD/1980/29/29007/pdf)

[31] Zaag H. Blow-up results for vector-valued nonlinear heat equation with no gradient structure, Ann. Inst. H. Poincaré Anal. Non Linéaire 15 (1998), 581-622. (MR1643389) (doi: 10.1016/S0294-1449(98)80002-4)

[32] Zakharov V.E. Collapse of Langmuir waves, Soviet Phys. JETP 35 (1972), 908-914. 